\title{
On the size of Diophantine $m$-tuples in imaginary quadratic number rings
}

\author{
Nikola Adžaga \\ Department of Mathematics, Faculty of Civil Engineering \\ University of Zagreb, Kačićeva 26, Zagreb, Croatia \\ nadzaga@grad.hr
}

Received 12 August 2019

Accepted 14 August 2019

Published 17 September 2019

Communicated by Efim Zelmanov

\begin{abstract}
A Diophantine $m$-tuple is a set of $m$ distinct integers such that the product of any two distinct elements plus one is a perfect square. It was recently proven that there is no Diophantine quintuple in positive integers. We study the same problem in the rings of integers of imaginary quadratic fields. By using a gap principle proven by Diophantine approximations, we show that $m \leq 42$. Our proof is relatively simple compared to the proofs of similar results in positive integers.
\end{abstract}

Keywords: Diophantine $m$-tuples; Diophantine approximation; Pell equations; rings of integers; Diophantine equations.

Mathematics Subject Classification: Primary: 11D09; Secondary: 11J68

\section{Introduction}

Diophantus of Alexandria was studying sets of rational numbers such that the product of any two distinct elements is one less than a perfect square. The natural question regarding these sets is their possible size. Throughout history, this question has remained interesting to number theorists. Through Fermat (who found an integer Diophantine quadruple $\{1,3,8,120\}$ ) and Euler (who found an infinite family of Diophantine quadruples in positive integers), to Baker and Davenport, who have proven the first result on the uniqueness of the extension of a Diophantine triple to a quadruple [1. To be more specific, using linear forms in logarithms, they have proven that, if $\{1,3,8, d\}$ is a Diophantine quadruple in positive integers,

This is an Open Access article published by World Scientific Publishing Company. It is distributed under the terms of the Creative Commons Attribution 4.0 (CC BY) License which permits use, distribution and reproduction in any medium, provided the original work is properly cited. 
then $d=120$. In other words, there is a unique extension to a quadruple (and this extension gives us an example which Fermat found). Let us note here that, when we talk about the extension of a set, we implicitly assume that the property of being a Diophantine $m$-tuple is retained. The work of Baker and Davenport has motivated two conjectures — each triple can be extended to a quadruple with a larger element in a unique way, and, in a weaker version, there is no Diophantine quintuple in positive integers. This weaker conjecture was recently proven [7, while the stronger conjecture remains open. At a similar time, in 4], it was found that there are infinitely many Diophantine sextuples in rational numbers. Generalizing results from integers to rationals can be very hard. On the other hand, it is reasonable to assume that the transition to analogous problems in other rings of integers will be easier. However, even in this ambient, there are not many results, e.g. we find less than 10 papers solving similar problems in the ring of Gaussian integers. We can highlight [2, 6], which deal with the extension of Diophantine triples from one-parameter families. Yet, due to the similarities of this ring with the usual ring of rational integers, we can expect that the similar results will hold and that one can prove them using similar techniques. The goal of this research is to answer how many elements can a Diophantine $m$-tuple in imaginary quadratic number ring have, or, to be more specific and modest, to provide an upper bound on the size of a Diophantine $m$-tuple.

Let $\mathcal{O}_{K}$ be the ring of integers of an imaginary quadratic number field $K$. A Diophantine triple $\{a, b, c\} \subseteq \mathcal{O}_{K}$ induces an elliptic curve $E: y^{2}=(a x+1)(b x+$ $1)(c x+1)$ defined over $K$. For every $d$ such that $\{a, b, c, d\}$ is Diophantine quadruple, there exist $r, s, t$ such that $a d+1=r^{2}, b d+1=s^{2}$ and $c d+1=t^{2}$. We obtain an $\mathcal{O}_{K^{-}}$ integral point $(d, r s t)$ on $E$. Since an elliptic curve has finitely many integral points [10], every Diophantine triple can be extended with only finitely many elements. We conclude that every Diophantine $m$-tuple is finite. Unfortunately, this does not provide us with an effective absolute bound on the size of such sets.

We approach this problem in the following way. Assume that a Diophantine triple $\{a, b, c\}$ in imaginary quadratic number ring $\mathcal{O}_{K}$ can be extended with a fourth element $d$. By eliminating $d$ from the equations it satisfies $\left(a d+1=x^{2}\right.$, $b d+1=y^{2}$ and $c d+1=z^{2}$ ), we get a system of two Pell-type equations with common unknown. A solution of this system gives us two simultaneous approximations of square roots close to 1 . Then, we apply a variant of Bennett's theorem developed for imaginary quadratic number rings by Jadrijević and Ziegler [8]. We obtain the gap principle which can be tersely stated as follows. In a Diophantine quadruple, if the third element (by size) is much bigger than the second element, then the fourth element is bounded from above by a power of the third element. Combining this result with a simple lower bound on the largest element of a Diophantine quadruple (in terms of the smallest element), we obtain a proof by contradiction (assuming that there exists a Diophantine $m$-tuple with $m \geq 43$ ). Thus, the main result of this paper is the following theorem. 
Theorem 1. There is no Diophantine m-tuple in imaginary quadratic number ring with $m \geq 43$.

\section{System of Pell-Type Equations}

Let $\{a, b, c\} \subset \mathcal{O}_{K}$ be a Diophantine triple in the imaginary quadratic number ring $\mathcal{O}_{K}$. Without loss of generality, we may assume $0<|a| \leq|b| \leq|c|$. Then there are $r, s$ and $t$ in $\mathcal{O}_{K}$ such that $a b+1=r^{2}, a c+1=s^{2}, b c+1=t^{2}$.

Since the equation $X^{2}-Y^{2}=1$ has only trivial solutions $(X Y=0)$, the numbers $a c$ and $b c$ are not squares in $\mathcal{O}_{K}$. Namely, $a c \neq i^{2}=-1$ and $|s| \neq 1$ since there are no three numbers $\{a, b, c\}$ of absolute value 2 or less which make up a Diophantine triple. Similarly, $a b$ is not a square: if we assume the contrary, then $r=0$ and $\{a, b\}=\{-1,1\}$ (or $\{a, b\}=\left\{\frac{-1+\sqrt{-3}}{2}, \frac{1+\sqrt{-3}}{2}\right\}$ ). There is no $c \neq 0$ such that $\{a, b, c\}$ is a Diophantine triple, because $-c+1=s^{2}$ and $c+1=t^{2}$ imply $1-c^{2}=s^{2} t^{2}$, hence $c=0$ or $s t=0$, which means $c= \pm 1 \in\{a, b\}$. Similar reasoning resolves the case $\{a, b\}=\left\{\frac{-1+\sqrt{-3}}{2}, \frac{1+\sqrt{-3}}{2}\right\}$, subtracting and multiplying equations containing $c$ shows that $c= \pm 1$, but $\{a, b, c\}$ is not a Diophantine triple.

Let us note here that the solutions of this equation $\left(X^{2}-Y^{2}=1\right)$ in the ring of integers of imaginary quadratic field are described in [5]. Numbers $a b, a c$ and $b c$ are not squares even in $K$ since $\mathcal{O}_{K}$ is integrally closed in $K$. We have proven the following lemma.

Lemma 1. If $\{a, b, c\}$ is a Diophantine triple in the imaginary quadratic number ring $\mathcal{O}_{K}$ and $a b c \neq 0$, then $a b$, ac and $b c$ are not squares in $K$.

If there is $d \in \mathcal{O}_{K}$ such that $\{a, b, c, d\}$ is a Diophantine quadruple, then there are $x, y, z \in \mathcal{O}_{K}$ such that $a d+1=x^{2}, b d+1=y^{2}, c d+1=z^{2}$. Therefore $a(c d+1)-c(a d+1)=a z^{2}-c x^{2}$ and $a z^{2}-c x^{2}=a-c$. Analogously, eliminating $d$ from the second and third equation, we get $b z^{2}-c y^{2}=b-c$. We have obtained a system of the equations

$$
\begin{gathered}
a z^{2}-c x^{2}=a-c, \\
b z^{2}-c y^{2}=b-c .
\end{gathered}
$$

These equations are similar to Pell's equations and their solutions have a very similar structure. The solutions of Pell-type equations $\left(x^{2}-D y^{2}=N\right)$ in imaginary quadratic rings are described in [5].

From now on, we will always assume that 0 is not an element of a Diophantine $m$-tuple. We will also assume that the elements are sorted by absolute value (in ascending order).

\section{Gap Principle (Obtained by Diophantine Approximations)}

Here, we prove that the solution of (11) and (2) provides us two simultaneous approximations of square roots close to 1 by elements of $K$. 
Lemma 2. Let $(x, y, z)$ be a solution of the system of equations (11) and (2). Assume that $|c|>4|b|$ and $|a| \geq 2$. If $\theta_{1}^{(1)}= \pm \frac{s}{a} \sqrt{\frac{a}{c}}, \theta_{1}^{(2)}=-\theta_{1}^{(1)}$ and $\theta_{2}^{(1)}=$ $\pm \frac{t}{b} \sqrt{\frac{b}{c}}, \theta_{2}^{(2)}=-\theta_{2}^{(1)}$, where the signs are chosen such that

$$
\left|\theta_{1}^{(1)}-\frac{s x}{a z}\right| \leq\left|\theta_{1}^{(2)}-\frac{s x}{a z}\right| \quad \text { and } \quad\left|\theta_{2}^{(1)}-\frac{t y}{b z}\right| \leq\left|\theta_{1}^{(2)}-\frac{t y}{b z}\right|
$$

then

$$
\begin{aligned}
& \left|\theta_{1}^{(1)}-\frac{s b x}{a b z}\right| \leq \frac{|s| \cdot|c-a|}{|a| \sqrt{|a c|}} \cdot \frac{1}{|z|^{2}}<\frac{21}{16} \frac{|c|}{|a|} \cdot \frac{1}{|z|^{2}} \text { and } \\
& \left|\theta_{2}^{(1)}-\frac{t a y}{a b z}\right| \leq \frac{|s| \cdot|c-b|}{|b| \sqrt{|b c|}} \cdot \frac{1}{|z|^{2}}<\frac{21}{16} \frac{|c|}{|a|} \cdot \frac{1}{|z|^{2}} .
\end{aligned}
$$

Proof. The following holds:

$$
\begin{aligned}
\left|\theta_{1}^{(1)}-\frac{s x}{a z}\right| & =\left|\left(\theta_{1}^{(1)}\right)^{2}-\frac{s^{2} x^{2}}{a^{2} z^{2}}\right| \cdot\left|\theta_{1}^{(1)}+\frac{s x}{a z}\right|^{-1} \\
& =\left|\frac{s^{2}}{a^{2}}\right| \cdot \frac{\left|a z^{2}-c x^{2}\right|}{|c||z|^{2}} \cdot\left|\theta_{1}^{(2)}-\frac{s x}{a z}\right|^{-1} \\
& =\frac{|s|^{2}|c-a|}{|a|^{2}|c|}\left|\theta_{1}^{(2)}-\frac{s x}{a z}\right|^{-1} \cdot \frac{1}{|z|^{2}} .
\end{aligned}
$$

Since $2\left|\theta_{1}^{(2)}-\frac{s x}{a z}\right| \geq\left|\theta_{1}^{(2)}-\frac{s x}{a z}\right|+\left|\theta_{1}^{(1)}-\frac{s x}{a z}\right| \geq\left|\theta_{1}^{(2)}-\frac{s x}{a z}-\left(\theta_{1}^{(1)}-\frac{s x}{a z}\right)\right|=$ $\left|\theta_{1}^{(2)}-\theta_{1}^{(1)}\right|=2\left|\frac{s}{a} \sqrt{\frac{a}{c}}\right|$, we conclude that $\left|\theta_{1}^{(2)}-\frac{s x}{a z}\right| \geq\left|\frac{s}{a} \sqrt{\frac{a}{c}}\right|$.

Hence $\left|\theta_{1}^{(1)}-\frac{s b x}{a b z}\right| \leq \frac{|s|^{2}|c-a|}{|a|^{2}|c|} \mid \frac{a}{s} \sqrt{\frac{c}{a} \mid \cdot \frac{1}{|z|^{2}}}$, which implies the first inequality in this lemma's statement. We also need to prove $\frac{|s| \cdot|c-a|}{|a| \sqrt{|a c|}} \cdot \frac{1}{|z|^{2}}<\frac{21}{16} \frac{|c|}{|a|} \cdot \frac{1}{|z|^{2}}$, i.e. $|\sqrt{a c+1}|$. $|c-a|<\frac{21}{16}|c| \sqrt{|a c|}$, which is equivalent to $\left|\sqrt{1+\frac{1}{a c}}\right|<\frac{21}{16} \frac{|c|}{|c-a|}$. The condition $|c|>4|a|$ implies that $\frac{21}{16} \frac{|c|}{|c-a|} \geq \frac{21}{20}$. Left-hand side is $\left|\sqrt{1+\frac{1}{a c}}\right| \leq \sqrt{\left|1+\frac{1}{|a c|}\right|} \leq$ $\sqrt{1+\frac{1}{16}}=\frac{\sqrt{17}}{4}$, implying the second inequality stated.

The other pair of inequalities is proven analogously (we have used $|c|>4|a|$ and $|a| \geq 2$ ).

We will now apply Jadrijević-Ziegler theorem from [8].

Theorem 2 (Jadrijević-Ziegler [8, Theorem 7.1]). Let $\theta_{i}=\sqrt{1+\frac{a_{i}}{T}}, i=1,2$ with $a_{1}$ and $a_{2}$ distinct quadratic integers in the imaginary quadratic field $K$ and let $T$ be an algebraic integer of $K$. Further, let $M=\max \left\{\left|a_{1}\right|,\left|a_{2}\right|\right\},|T|>M$ and

$$
L=\frac{27}{16\left|a_{1}\right|^{2}\left|a_{2}\right|^{2}\left|a_{1}-a_{2}\right|^{2}}(|T|-M)^{2}>1 .
$$


Then

$$
\max \left\{\left|\theta_{1}-\frac{p_{1}}{q}\right|,\left|\theta_{2}-\frac{p_{2}}{q}\right|\right\}>c|q|^{-\lambda},
$$

for all algebraic integers $p_{1}, p_{2}, q \in K$, where

$$
\begin{aligned}
& \lambda=1+\frac{\log P}{\log L}, \quad c^{-1}=4 p P(\max \{1,2 l\})^{\lambda-1}, \\
& l=\frac{27}{64} \frac{|T|}{|T|-M}, \quad p=\sqrt{\frac{2|T|+3 M}{2|T|-2 M}}, \\
& P=16 \frac{\left|a_{1}\right|^{2}\left|a_{2}\right|^{2}\left|a_{1}-a_{2}\right|^{2}}{\min \left\{\left|a_{1}\right|,\left|a_{2}\right|,\left|a_{1}-a_{2}\right|\right\}^{3}}(2|T|+3 M) .
\end{aligned}
$$

Using this theorem, we will show that, if the second and the third element of a Diophantine $m$-tuple are sufficiently away from each other, then the fourth element is bounded by a power of the third element. More precisely, we prove the following proposition.

Proposition 1 (Gap Principle). If $\{a, b, c, d\} \subseteq \mathcal{O}_{K}$ is a Diophantine quadruple such that $|a c| \geq 9,|b| \geq \frac{3}{2}|a|,|b|>5$ and $|c|>|b|^{15}$, then $|d|<4278^{20}|c|^{50}$.

Proof. The solution $(x, y, z)$ of (1) and (2) gives us simultaneous approximations of

$$
\theta_{1}= \pm \frac{s}{a} \sqrt{\frac{a}{c}}= \pm \sqrt{\frac{s^{2} a}{a^{2} c}}= \pm \sqrt{\frac{a c+1}{a c}}= \pm \sqrt{1+\frac{b}{a b c}} \text { and } \theta_{2}= \pm \sqrt{1+\frac{a}{a b c}}
$$

We let $a_{0}=0, a_{1}=b, a_{2}=a, T=a b c, M=|b|$, and note that $l=\frac{27|a b c|}{64(|a b c|-|b|)}<\frac{1}{2}$, since this is equivalent to $27|a c|<32(|a c|-1)$, which holds for $|a c|>\frac{32}{5}$. Similarly,

$$
p=\sqrt{\frac{2|a b c|+3|b|}{2|a b c|-2|b|}}=\sqrt{1+\frac{5}{2(|a c|-1)}} \leq \sqrt{1+\frac{5}{2(9-1)}}=\sqrt{\frac{21}{16}} .
$$

Since $l<\frac{1}{2}$, it follows that $c=\frac{1}{4 p P}$. Hence, by (3), $c \geq \frac{1}{\sqrt{21} P}$. Hence

We also observe that $\min \{|a|,|b|,|b-a|\} \geq \frac{|a|}{2}$ because $|b-a| \geq|b|-|a| \geq \frac{|a|}{2}$.

$$
\begin{aligned}
& P=16 \frac{|a|^{2}|b|^{2}|b-a|^{2}}{\min \{|a|,|b|,|b-a|\}^{3}}(2|a b c|+3|b|) \leq 128 \frac{|b|^{3}|b-a|^{2}}{|a|}(2|a c|+3) \quad \text { and } \\
& L=\frac{27}{16|a|^{2}|b|^{2}|b-a|^{2}}(|a b c|-|b|)^{2}=\frac{27(|a c|-1)^{2}}{16|a|^{2}|b-a|^{2}}
\end{aligned}
$$

Here, the condition $L>1$ of Jadrijević-Ziegler theorem is equivalent to $27(|a c|-$ $1)^{2}>16|a|^{2}|b-a|^{2}$. Taking the square root, we get a simpler claim $3 \sqrt{3}(|a c|-1)>$ $4|a||b-a|$. Since $|c|>|b|^{3}$ (and $\left.|b| \geq \frac{3}{2}|a|\right)$, even a stronger claim, $|a c|-1>|a||b-a|$, 
holds. Namely, using the assumptions and the triangle inequality we get $|a c|-1>$ $|a| \cdot|b|^{3}-1>2|a|^{2}|b|-1>|a| \cdot|b|+\left|a^{2}\right| \geq\left|a b-a^{2}\right|=|a||b-a|$.

We observe that $\lambda>1$ since both $P$ and $L$ are greater than $1(|b|>|a|$ implies $P>1$ ). We will show that $|c|>|b|^{15}$ implies $\lambda<1$.9. Since $\lambda=1+\frac{\log P}{\log L}$, we obtain the equivalent statements $\lambda<1.9, \frac{\log P}{\log L}<0.9$, and $P<L^{0.9}$. Plugging $L$ and using the inequality proven for $P$, we see that we should prove

$$
128|b|^{3}|b-a|^{3.8}|a|^{0.8}(2|a c|+3)<\left(\frac{27}{16}\right)^{0.9}(|a c|-1)^{1.8} .
$$

Since $|a c|-1>\frac{8}{21}(2|a c|+3)$, it suffices to show that $336|b|^{3}|b-a|^{3.8}|a|^{0.8}<$ $\left(\frac{27}{16}\right)^{0.9}(|a c|-1)^{0.8}$. We prove the stronger inequality

$$
210|b|^{3}|b-a|^{3.8}|a|^{0.8}<(|a c|-1)^{0.8} .
$$

The right-hand side of (44), $(|a c|-1)^{0.8}$ is greater than $(|a c|)^{0.8}-1$, because the function $f(t)=(t-1)^{0.8}-t^{0.8}+1$ is 0 at $t=1$ and the function is increasing. Hence, if $|c|>|b|^{15}$, then $(|a c|-1)^{0.8}>|a|^{0.8}|b|^{12}-1$, which is greater than the left-hand side of (4), $210|b|^{3}|b-a|^{3.8}|a|^{0.8}$, because of the larger degree of $|b|$. More precisely, since $|b| \geq \frac{3}{2}|a|$, i.e. $|a| \leq \frac{2}{3}|b|$, we conclude that

$$
210|b|^{3}|b-a|^{3.8}|a|^{0.8} \leq 210|b|^{3}\left(\frac{5}{3}|b|\right)^{3.8}\left|\frac{2 b}{3}\right|^{0.8}<1058|b|^{7.6}<|b|^{12}-1 .
$$

The last inequality is again obtained by simple analysis of auxiliary function $f(t)=$ $t^{12}-1058 t^{7.6}-1$ whose largest root 4.86836 is determined numerically. Since $|b|>5$, we have proven that $\lambda<1.9$.

Jadrijević-Ziegler theorem, together with Lemma 2 yields

$$
\frac{21}{16} \frac{|c|}{|a|} \cdot \frac{1}{|z|^{2}}>\frac{1}{\sqrt{21} P}|a b z|^{-\lambda} \geq \frac{|a|}{\sqrt{21} \cdot 128|b|^{3}|b-a|^{2}(2|a c|+3)}|a b z|^{-\lambda},
$$

implying $168 \sqrt{21} \frac{|c|}{|a|^{2}}|b|^{3}|b-a|^{2}(2|a c|+3) \cdot|a b|^{\lambda}>|z|^{2-\lambda}>|z|^{0.1}$. Therefore,

$$
\begin{aligned}
|z|^{0.1} & <168 \sqrt{21}|c| \cdot 3|a c| \cdot|b-a|^{2}|b|^{3+\lambda}|a|^{\lambda-2} \\
& <504 \sqrt{21}|c|^{2} \cdot \frac{2}{3}|b| \cdot\left(\frac{5}{3}|b|\right)^{2}|b|^{4.9} \quad(\text { since }|b| \geq 2|a| \text { and } \lambda<1.9) \\
& <4728|c|^{2}|b|^{7.9}<4728|c|^{\frac{30+7.9}{15}}<4728|c|^{2.53}
\end{aligned}
$$

and, finally, $|z|<4728^{10}|c|^{2.53 \cdot 10}=4728^{10}|c|^{25.3}$.

We conclude $|d|=\frac{\left|z^{2}-1\right|}{|c|} \leq \frac{|z|^{2}+1}{|c|} \leq \frac{4728^{20}|c|^{50.6}+1}{|c|}<4728^{20}|c|^{50}$. 


\section{A Lower Bound on the Element Extending a Diophantine Triple}

In the previous section, we have proved an upper bound on the fourth element of a Diophantine $m$-tuple. We will now find a lower bound so that we can juxtapose these two bounds. To find it, we need a definition and a few lemmas.

Definition 1. A Diophantine triple $\{a, b, c\}$ is regular if $c=a+b \pm 2 r$, where $r^{2}=a b+1$.

Lemma 3. If $\{a, b, c, d\}$ is a Diophantine quadruple such that $2 \leq|a| \leq|b| \leq|c| \leq$ $|d|$, then at least one of the triples $\{a, b, c\}$ and $\{a, b, d\}$ is not regular, i.e. it is impossible that $c=a+b-2 r$ and $d=a+b+2 r$ (or vice versa), where $a b+1=r^{2}$.

Proof. Assume the contrary. Then $c d=(a+b)^{2}-4 r^{2}=a^{2}+2 a b+b^{2}-4(a b+1)=$ $a^{2}-2 a b+b^{2}-4$ and $c d+1=(a-b)^{2}-3$. Since $\{c, d\}$ is a Diophantine pair, there is an integer $z \in \mathcal{O}_{K}$ such that $c d+1=z^{2}$. Hence $(a-b)^{2}-3=z^{2}$, i.e. $(a-b-z)(a-b+z)=3$. Therefore, on the left-hand side there are two elements of $\mathcal{O}_{K}$ of absolute value less than 3 (or one of them is a unit) and their norms are divisors of 9 .

First, we deal with non-unit solutions in the case $K=\mathbb{Q}[\sqrt{D}]$, where $D \equiv 1$ $(\bmod 4)$. Let $\rho=\frac{-1+\sqrt{D}}{2}$ be the generator of $\mathcal{O}_{K}$ over $\mathbb{Z}$. Using the fact that the absolute value of the norm of $x+y \rho$ is $\left|x^{2}+x y-\frac{D-1}{4} y^{2}\right|=\left(x+\frac{y}{2}\right)^{2}+\frac{|D|}{4} y^{2}=3$, we easily see that for $|D| \geq 13, y$ must be 0 and $x+\frac{y}{2}= \pm \sqrt{3}$, which is impossible. For $D=-7$, multiplying the equation by 4 , we get $(2 x+y)^{2}+7 y^{2}=12$, which has no solutions in integers $x$ and $y$. For $D=-3$ we get the equation $(2 x+y)^{2}+$ $3 y^{2}=12$ which has solutions $x=-1, y=2$ and $x=1, y=-2$. This gives us $a-b-z=-1+2 \rho, a-b+z=1-2 \rho$ (or vice versa) and $c d=z^{2}-1=-4 \sqrt{-3}$ (or $c d=-4$ ), which implies that $|c| \leq \sqrt{4 \sqrt{3}}$. Now, we check all triples with absolute value of elements between 2 and $\sqrt{4 \sqrt{3}}$ to find only two Diophantine triples $\{-2,2,-2 \sqrt{-3}\}$ and $\{-2,2,2 \sqrt{-3}\}$. However, $-2 \sqrt{-3} \cdot 2 \sqrt{-3}+1=13$ is not a square, so $\{a, b, a+b-2 r, a+b+2 r\}=\{-2,2,-2 \sqrt{-3}, 2 \sqrt{-3}\}$ is not a Diophantine quadruple.

Now, let $K=\mathbb{Q}[\sqrt{D}]$ where $D \equiv 2,3(\bmod 4)$. Analogously as in the previous case, $x^{2}+|D| y^{2}<3$ has no non-unit solutions for $|D| \geq 6$. Similarly, for $D=-2$, we get the solution $a-b \pm z=1 \pm \sqrt{-2}$, which implies $2 z= \pm 2 \sqrt{-2}$, i.e. $c d=-3$. However, one checks that there is no Diophantine quadruple in $\mathbb{Z}[\sqrt{-2}]$ with such small elements (and $|a| \geq 2$ ).

Unit solutions are easy to check, e.g. in $\mathbb{Z}[i]$ number 3 is prime, and it can be factored in $\mathbb{Z}[i]$ in the following ways: $3=1 \cdot 3,3=-1 \cdot(-3), 3=i \cdot(-3 i), 3=-i \cdot 3 i$, up to the order of the factors. In first two cases, subtracting $a-b+z$ and $a-b-z$ implies $2 z= \pm 2$, i.e. $z= \pm 1$, which would imply $c d=0$. In the other two cases, we conclude $z= \pm 2 i$ in the same manner, which implies $c d+1=-4$ and $c d=-5$, which is impossible for $|c|>\sqrt{5}$. Now, one just checks that there is no Diophantine 
triple $\{a, b, c\}$ such that $2 \leq|a|,|b|,|c| \leq \sqrt{5}$. Analogous reasoning resolves the number $\operatorname{ring} \mathcal{O}_{K}=\mathbb{Z}\left[\frac{-1+\sqrt{-3}}{2}\right]$.

Lemma 4. If $\{a, b, c, d\}$ is a Diophantine quadruple such that $2 \leq|a| \leq|b| \leq|c| \leq$ $|d|$, then $|d| \geq \frac{|a b|}{8} \geq \frac{|a|^{2}}{8}$.

Remark 1. We conjecture that the stronger claim also holds, if $d \neq a+b+c+$ $2 a b c \pm 2 r s t$ (where $s^{2}=a c+1$ and $t^{2}=b c+1$ ), then $|d| \geq 4|a b|$. This claim holds in $\mathbb{Z}$ (see, e.g. 9]).

Proof. Using the previous lemma, without loss of generality, we may assume that $\{a, b, d\}$ is not a regular triple. Denote $a b+1=r^{2}, a d+1=x^{2}, b d+1=y^{2}$.

Then $c_{ \pm}=a+b+d+2 a b d \pm 2 r x y$ is not 0 . Indeed, the claim $c_{ \pm}=0$ is equivalent to the claims $a+b+d+2 a b d=\mp 2 r x y,(2 a b+1)^{2} d^{2}+2(a+b)(2 a b+1) d+(a+b)^{2}=$ $4(a b+1)(a d+1)(b d+1)$ and $d^{2}-2(a+b) d+(a-b)^{2}-4=0$. Solutions of this quadratic equation for $d$ are exactly $a+b \pm 2 r$. Since $\{a, b, d\}$ is not a regular triple $(d \neq a+b \pm 2 r)$, we conclude that $c_{ \pm} \neq 0$.

Consider now the product $c_{+} c_{-}=(a+b+d+2 a b d)^{2}-4 r^{2} x^{2} y^{2}=a^{2}+b^{2}+$ $d^{2}-2 a b-2 a d-2 b d-4$. Hence $\left|c_{+} c_{-}\right| \leq|a|^{2}+|b|^{2}+|d|^{2}+2|a b|+2|a d|+2|b d|+4$. Since $|d| \geq|a|,|b|$ and $|d|^{2} \geq 4$ (there is no Diophantine quadruple with the largest element less than 2 by absolute value), it follows that $\left|c_{+} c_{-}\right| \leq 10|d|^{2}$.

On the other hand, $\left|c_{+}+c_{-}\right|=2|a+b+d+2 a b d|$. Assume that $\left|c_{+}\right| \geq\left|c_{-}\right|$. Then $2\left|c_{+}\right| \geq\left|c_{+}\right|+\left|c_{-}\right| \geq\left|c_{+}+c_{-}\right|$and $\left|c_{+}\right| \geq|a+b+d+2 a b d|$.

Since $|b| \geq|a| \geq 2$, it follows that $|a b| \geq 4$, so $|a+b+d| \leq 3|d| \leq \frac{3}{4}|a b d|$. We conclude that

$$
\left|c_{+}\right| \geq|a+b+d+2 a b d| \geq 2|a b d|-|a+b+d| \geq 2|a b d|-\frac{3}{4}|a b d|=\frac{5}{4}|a b d| .
$$

Juxtaposition of this lower bound on $\left|c_{+}\right|$with the upper bound on $\left|c_{+} c_{-}\right|$implies that $\left|c_{-}\right| \leq \frac{10|d|^{2}}{\left|c_{+}\right|} \leq \frac{10|d|^{2}}{\frac{5}{4}|a b d|}=\frac{8|d|}{|a b|}$. Because $c_{-} \neq 0$, it follows that $\left|c_{-}\right| \geq 1$, so we can conclude that $\frac{8|d|}{|a b|} \geq\left|c_{-}\right| \geq 1$ and $|d| \geq \frac{|a b|}{8} \geq \frac{|a|^{2}}{8}$.

\section{An Upper Bound on the Size of Diophantine $m$-Tuple}

Here, we prove our main result, the following theorem.

Theorem 1, There is no Diophantine m-tuple in imaginary quadratic number ring $\mathcal{O}_{K}$ with $m \geq 43$.

Proof. Assume the contrary, that there is a Diophantine $m$-tuple $\left\{a_{1}, a_{2}, \ldots, a_{m}\right\}$ sorted by absolute value $\left(0<\left|a_{1}\right| \leq \cdots \leq\left|a_{m}\right|\right)$ and $m \geq 43$. By using the computer, we have checked that there is no Diophantine quintuple with absolute value of elements at most 16 . For a fixed $D$ it is clear that one can do this. For $|D|>32$ all the elements of absolute value less or equal than 16 are real ones. On 
the other hand, for $|D|>256$, it is not possible that $a_{i} a_{j}+1=(y \sqrt{-D})^{2}=-D y^{2}$ since $\left|a_{i} a_{j}+1\right| \leq 257$. Therefore, $\left|a_{4}\right| \geq 2,\left|a_{5}\right| \geq 16$. Now, we repeatedly apply Lemma 4 on different subsets of $\left\{a_{1}, a_{2}, \ldots, a_{m}\right\}$, and each obtained inequality is used in the next appliance:

$$
\begin{gathered}
\left\{a_{7}, a_{8}, a_{9}, a_{10}\right\} \stackrel{\text { Lemma }}{\Longrightarrow}\left|a_{10}\right| \geq \frac{\left|a_{7}\right|^{2}}{8} \\
\left\{a_{10}, a_{11}, a_{12}, a_{13}\right\} \Rightarrow\left|a_{13}\right| \geq \frac{\left|a_{10}\right|^{2}}{8} \geq \frac{\left|a_{7}\right|^{4}}{8^{3}} \\
\left\{a_{13}, a_{14}, a_{15}, a_{16}\right\} \Rightarrow\left|a_{16}\right| \geq \frac{\left|a_{13}\right|^{2}}{8} \geq \frac{\left|a_{7}\right|^{8}}{8^{7}} \\
\vdots \\
\left\{a_{22}, a_{23}, a_{24}, a_{25}\right\} \Rightarrow\left|a_{25}\right| \geq \frac{\left|a_{7}\right|^{64}}{8^{63}} .
\end{gathered}
$$

Let us now show that we can apply Proposition 1 on $\left\{a_{4}, a_{7}, a_{25}, a_{25+k}\right\}$ for $k>0$ since $\left|a_{4}\right| \geq 2$ and $\left|a_{5}\right|>12$. Namely, by using Lemma 4 on $\left\{a_{4}, a_{5}, a_{6}, a_{7}\right\}$, we can conclude that $\left|a_{7}\right| \geq \frac{\left|a_{4} a_{5}\right|}{8}>\frac{3}{2}\left|a_{4}\right|$. Previously obtained inequality guarantees that $\left|a_{25}\right|>\left|a_{7}\right|^{15}$ (it suffices to show that $\frac{\left|a_{7}\right|^{64}}{8^{63}}>\left|a_{7}\right|^{15}$, i.e. $\left|a_{7}\right|>14.5$, which holds because $\left|a_{7}\right| \geq\left|a_{5}\right| \geq 16$ ). The conditions of Proposition 1 hold.

Therefore, Proposition 1 implies

$$
\left|a_{25+k}\right|<4278^{20}\left|a_{25}\right|^{50} \text {. }
$$

However, we can continue to apply Lemma 4.

$$
\begin{aligned}
\left\{a_{25}, a_{26}, a_{27}, a_{28}\right\} & \Rightarrow\left|a_{28}\right| \geq \frac{\left|a_{25}\right|^{2}}{8} \\
\left\{a_{28}, a_{29}, a_{30}, a_{31}\right\} & \Rightarrow\left|a_{31}\right| \geq \frac{\left|a_{28}\right|^{2}}{8} \geq \frac{\left|a_{25}\right|^{4}}{8^{3}} \\
\ldots & \Rightarrow\left|a_{43}\right| \geq \frac{\left|a_{25}\right|^{64}}{8^{63}}>4278^{20}\left|a_{25}\right|^{50},
\end{aligned}
$$

which contradicts the inequality (5). Namely, the last inequality is equivalent to $\left|a_{25}\right|^{14} \geq 8^{63} \cdot 4278^{20}$, i.e. it is true for $\left|a_{25}\right|>1.784 \cdot 10^{9}$, which holds since $\left|a_{25}\right| \geq$ $\frac{\left|a_{7}\right|^{64}}{8^{63}} \geq \frac{16^{64}}{8^{63}}$.

Let us note here that computer search did not yield any Diophantine quintuple in imaginary quadratic numbers rings, nor has the more systematic search by Gibbs found Diophantine quintuples in $\mathbb{Z}[\sqrt{-d}]$ for positive integer $d<50$ (as reported on the ResearchGate). Even so, we do not see any a priori reason why would all the imaginary quadratic number rings have the largest Diophantine $m$-tuple of the same size $m$. The method used here most likely will not suffice to prove the strongest upper bound, even in the more specific situation of Gaussian integers. However, it is interesting that we have managed to find such a simple proof of the first uniform 
bound on the size of Diophantine $m$-tuple (in imaginary quadratic number rings) because such results usually required more complex proofs involving linear forms in logarithms (see, for example, [3, for the first proof of the uniform upper bound in integers).

\section{Acknowledgments}

The author would like to thank Andrej Dujella, Alan Filipin and Zrinka Franušić for motivating this research and suggesting numerous improvements to both the proof and the manuscript text.

This work was supported by the Croatian Science Foundation under the Project No. 6422 .

\section{References}

[1] A. Baker and H. Davenport, The equations $3 x^{2}-2=y^{2}$ and $8 x^{2}-7=z^{2}$, Quart. J. Math. Oxford Ser. (2) 20(2) (1969) 129-137.

[2] A. Bayad, A. Filipin and A. Togbé, Extension of a parametric family of Diophantine triples in Gaussian integers, Acta Math. Hungar. 148(2) (2016) 312-327.

[3] A. Dujella, An absolute bound for the size of Diophantine $m$-tuples, J. Number Theory 89 (2001) 126-150.

[4] A. Dujella, M. Kazalicki, M. Mikić and M. Szikszai, There are infinitely many rational Diophantine sextuples, Int. Math. Res. Not. IMRN 2017(2) (2017) 490-508.

[5] L. Fjellstedt, On a class of Diophantine equations of second degree in imaginary quadratic fields, Ark. Mat. 2(24) (1953) 435-461.

[6] Z. Franušić, On the extensibility of Diophantine triples $\{k-1, k+1,4 k\}$ for Gaussian integers, Glas. Mat. Ser. III 43(2) (2008) 265-291.

[7] B. He, A. Togbé and V. Ziegler, There is no Diophantine quintuple, Trans. Amer. Math. Soc. 371 (2019) 6665-6709.

[8] B. Jadrijević and V. Ziegler, A system of relative Pellian equations and a related family of relative Thue equations, Int. J. Number Theory 2(4) (2006) 569-590.

[9] B. W. Jones, A second variation on a problem of Diophantus and Davenport, Fibonacci Quart. 16 (1978) 155-165.

[10] C. L. Siegel, Uber einige anwendungen diophantischer approximationen, On Some Applications of Diophantine Approximations: A Translation of Carl Ludwig Siegel's Über Einige Anwendungen Diophantischer Approximationen by Clemens Fuchs, With a Commentary and The Article Integral Points on Curves: Siegel's Theorem After Siegel's Proof by Clemens Fuchs and Umberto Zannier, ed. U. Zannier (Pisa, Edizioni della Normale, 2014), pp. 81-138. 